\title{
Research on the Brand Strategy of SexyTea
}

\author{
Yifan Hao \\ Ocean University of China, College of Literature and Journalism, Qingdao, China,266100 \\ Author's Email: hyfshenqing123@163.com
}

\begin{abstract}
Because of the low barriers for entering the tea drink, competition is fierce, thousands of regional tea brands flourish and then fall before they accomplished the expansion in a short period. The giants in the tea drink industry always keep a steady increase. It seems that the market of tea drink is saturated, but SexyTea broke the rule by using unique expansion strategies and clever digital marketing strategies. Comparing to other giants in the tea drink industry, SexyTea chose a completely different way to survive. Without taking market share of several metropolis in China, SexyTea focused on the quality of drinkings and started expansion in Changsha City. With the help of digital marketing and recommendation from KOIs, SexyTea has been considered as a representative of Changsha city and buying a SexyTea has become a trend for tourists. With special pricing and direct-sale store module, SexyTea maintained a good relationship with their customers and also attracted more potential customers by this unique service. Thus, SexyTea, a regional tea brand, only located in one city, became a well-known brand. After five years of preparation, SexyTea started to open a new branch outside Changsha. They chose the closest capital city, Wuhan, in 2020. The purpose of this paper is to add new ideas for the tea drink industry about promotion strategy and distribution by citing specific and featured activities.
\end{abstract}

Keywords: Tea drink, Brand strategy, Digital marketing, Brand image

\section{INTRODUCTION}

Tea drink is a modifying beverage including concentrated tea liquid used different extraction methods and fresh milk, finest cream or various fruits[1]. The emergence of tea drinks changes the beverage industry in China by creating a new category in recent years. A commonly accepted development of tea drinks had three consecutive stages. The first stage is 'Powder Era'(1990-1995) The second stage, 'Street Era' (1995-2015), was shared a similar feature of low-quality of materials. They mostly blended milk powder with fruit flavor addictive. The third stage 'tea drink Era' (2015 to present), select high-quality tea materials and innovative extraction methods supplemented by natural milk, fruits, etc[1]. Recent developments have revealed that there were 340 million people purchasing tea drink in 2020 and the figure will serge as expected. And the market volume will exceed 100 billion at the end of 2020[2].

Recent studies, from macro perspectives. have focused on the development blueprint of the tea drink industry. However, from micro perspectives, few studies concentrate on a very leading corporation. Some studies believed differentiated marketing and fulfill the requirements of customers can be considered as the key of SexyTea[3]. Other studies have analyzed the brand strategy of SexyTea through $4 p$ module, but the previous studies ignored the true distinguishing points of SexyTea. It is unfounded that citing simple and universal promotion to illustrate SexyTea attached importance to digital marketing[4]. In addition, this paper will also adopt the $4 \mathrm{p}$ model as a research method to illustrate how SexyTea spring up. 


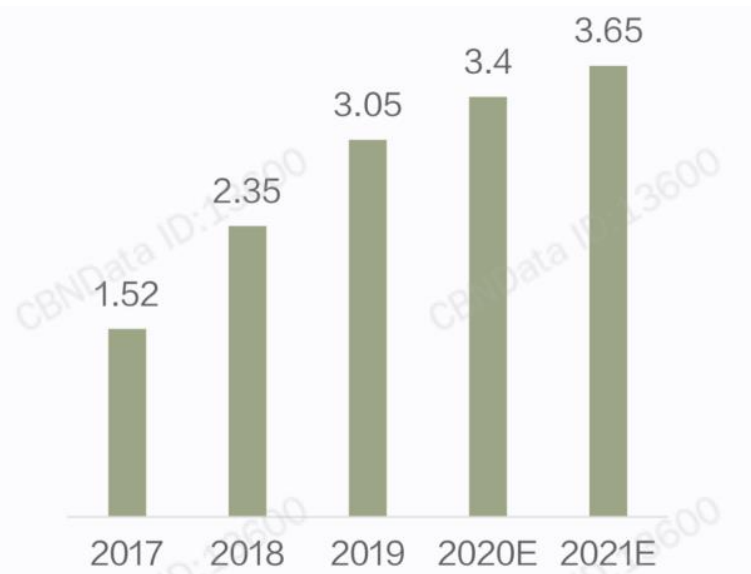

Figure 1. The consumers of tea drink from 2017 to 2019 and expected in 2020 and 2021[2]

\section{PRICE}

As table 1 shown, the price of SexyTea ranged from 10 yuan to 18 yuan, which is a medium level among other tea companies. The top 2 companies in the tea industry intended to set a higher price (13yuan to $35 y u a n)$, but more companies chose a similar pricing strategy (about10yuan to 20yuan). The reason why these companies set a similar pricing strategy is that $74.6 \%$ of tea consumers choose to purchased products in the range of 10 to 20 yuan[5]. Similar pricing strategy although makes SexyTea involve in a more fierce competitive environment, and this market volume is bigger than that of other parts. For most customers, the price is no longer a hinder of purchase and the customers do have much more choice in the same price range. Moreover, the discounts are also pricing. SexyTea mainly uses two types of discount, one is recharging membership card and give out bonus, the other one is directly using half price for the second cup on Wednesday or rainy day. By using this pricing strategy, SexyTea increase the revenue and harvest a reputation of high-quality with inexpensive price

Table 1 The pricing of main brands in the tea drink industry[2]

\begin{tabular}{llll}
\hline Brands & Establish & Main Product & Price Range \\
\hline NAYUKI & 2015 & Tea Drink \& Bread & 13-35 yuan \\
Hey Tea & 2012 & Tea Drink & 13-35 yuan \\
GoodMe Tea & 2010 & Tea Drink & 10-20 yuan \\
Zain Tea & 2019 & Tea Drink & 12-19 yuan \\
Honey Snow City & 1997 & Tea Drink & 5-10 yuan \\
Sweet 7 & 2015 & Tea Drink & $15-25$ yuan \\
Auntea Jenny & 2013 & Tea Drink & $10-20$ yuan \\
\hline
\end{tabular}

\section{PRODUCT}

SexyTea set a rule about quality to their consumers, which is permanent claim for a cup of fresh tea. If customers did not enjoy the flavor of drink or they found that the drink they ordered was of poor quality, they can get another fresh tea with the receipt. The customers who bought the drink were given a right that they can get another cup of drink for free for any reason. It means that free fresh tea service is an additional product of each drink they sold. Two cups of drink are only sold at one cup's price.

To a business extent, the free drinks service may cause a huge increase in costs, and the benefit is greatly reduced. It seems that this unwise service is risky for a beverage company with a large amount of customers. Conversely, the consumers feel the sincerity of company. Few consumers use the right to get another free drink and most customers become more and more trust Sexy
Tea. Sexy Tea make the special service as a product as well, and the buyers are willing to choose the Sexy Tea for this reason.

Besides the special service above, Sexy Tea also public the Monthly Food Safety Report through social media. The food safety checking originates from a food safety incident several years ago. Food safety agency found excessive levels of caffeine and sugar in some products of Sexy Tea. After apologizing to the public, SexyTea also established a monthly food safety checking to control the drink's quality. The checking report includes self-examination and consumer complaints. The public can see some slight problems such as tea dirt in the tea bucket, and also can see the corrective measures. The transparency of food safety improves the quality of SexyTea, and also brings the confidence of consumers. 


\section{PROMOTION}

Digital marketing is the component of marketing that utilizes the internet and online based digital technologies such as desktop computers, mobile phones and other digital media and platforms to promote products and services[6,7]. SexyTea now has opened an official account on the platform of Weibo, Wechat, TikTok, which are welcomed by all-aged people especially the youth. And SexyTea covers the main social media platform to build an online media matrix and release the latest information and campaigns of SexyTea. Then they identify a different feature of this platform, and releases various articles or pictures by using each feature to reach an effective of spreading. The number of followers in each channel has reached 100,000 , particularly the number of Weibo followers has reached 270,000 by now. But the number is still growing, more and more target customers witness their willingness to buy a cup of SexyTea on Weibo.

\subsection{Close Relation with Tourism}

At first, SexyTea did not have the ambition to intertwine with regional tourism. The reason why only focusing on one region is the lack of funds to open branches in other cities. From SexyTea's social account released in 2018, at present, the capacity is limited and only directly operated stores are opened in Changsha. But it remained a sense of marketing among these words, someone believes that certain promotion can be considered as regional hunger marketing[8]. Whatever it was, the effect of such promotion results in a surprising idea. Young tourists traveled to Changsha, Hunan province, intended to post the photos online through social media. The photos related to Changsha scenery and specials were spread to the public, after knowing that SexyTea only open stores in Changsha, the public spontaneously regarded SexyTea as one of the specials of Changsha. Especially, internet influencers or KOLs promoted the local brand on their channel and increased visibility. Then, the product of SexyTea was built a close relationship with tourism. The SexyTea official did not expect such relationship, but the word-of-mouth effect through the internet creates a brand new marketing strategy for SexyTea. Now, the travelers who visited Changsha must wait in lines and buy a cup of SexyTea. SexyTea maintained the local customers by their great service and attract travelers by reputations.

\subsection{Popularity of Brand Image}

Welcome and approachable image is formed in the minds of SexyTea's target customers. Brand image is the current view of the customers about a brand. It is an accumulation of contact and observation by people external to an organization[9].

About the brand image, there are brand visual designs, slogan and communication with customers involved. The brand visual design is unique, the most distinctive label that distinguishes SexyTea from other tea brands is cultural attributes. For example, the creativity of their cups often comes from famous paintings, and their new Chinese-style experimental series of concept stores widely use traditional culture. SexyTea successively bought the copyright of the court painter Castiglione and other famous paintings for a total of millions of dollars. Not only did SexyTea draw pictures of thousands of miles of rivers and mountains on the cups, but the posters of the stores were also antiques[8]. All these unique designs differentiate SexyTea from other brands, consumers believe that the traditional Chinese style is aesthetic and elegant. Secondly, an interview from the CEO of SexyTea, Lv Liang, said they identified the service as the core of SexyTea, and they want to create a classic brand with warmth. SexyTea not only launches the slogan but follows the slogan mentioned. People read the interview and formed a conception that SexyTea is trusted and welcomed. SexyTea does a great job on create storylines of the growth of SexyTea. According to a different platform, the editor creates various characters. For instance, more affable and approachable on Weibo, SexyTea always joins the other topic by using humorous words and style. They post their office joke to social media every day, and the employees who often appear in Weibo have all been nicknamed, and they have built up an image of enthusiasm and sincerity. The employees who run the Weibo account create a real working atmosphere. They also have tired time and joy of success. Thus, such a life that is true and close to ordinary people is conducive to the recognition of the public. After following their account, customers have a sense of familiarity, and they intend to recognize SexyTea as a friend from the web.

\section{PLACE}

\subsection{The Density of Direct-sale Store in Changsha}

SexyTea has spent 7 years developing itself in one city, and they opened 310 stores on $11819 \mathrm{~km} 2$ of land The density of stores in Changsha is high, and the number of stores is much exceeding their competitors. 
Table 2 The direct-sale store density of several brands in some cities.

\begin{tabular}{l|l|r|r|r|}
\hline brand name & city & city size $(\mathrm{km} 2)$ & number of stores & density \\
\hline Heytea & Beijing & 16410 & 62 & 0.0037 \\
\hline Heytea & Shanghai & 6340 & 101 & 0.0159 \\
\hline Nayuki & Beijing & 16410 & 23 & 0.0014 \\
\hline Nayuki & Shanghai & 6340 & 32 & 0.0051 \\
\hline SexyTea & Changsha & 11819 & 310 & 0.0262
\end{tabular}

It could be learned in Table 2 that the high density in Changsha firstly squeezes the development space of competitors, such as the other two giants only opened about 6 branches in Changsha. The market of tea drink in Changsha has been totally occupied by SexyTea. Secondly, SexyTea still taps the local market potential. In 2020, they opened nearly 130 stores in Changsha. There was a joke about SexyTea in Changsha that said the two SexyTea stores in Changsha only distance 3 meters. The most location of SexyTea is in the bustling shopping district, and the aggregation effect can attract customers' eyes then make them think of SexyTea.

\subsection{The Advantages of Direct-sale Store Module}

As mentioned above, the branches of SexyTea are all direct-sale stores. No franchise and no agent module benefit the control and management of company. The advantage of direct-sale module directly controlls every store and can timely and accurately implement the company's marketing philosophy, guidelines and policies. On the basis of SexyTea established raw material supply chain, the material is supplied by the headquarters and guarantee the standard is consistent. It reduces the possibility of regional inconsistency in flavor, and keeps a good quality.

The other advantage is companies directly facing consumers and understanding the characteristics of consumers' needs will help brand companies obtain the most effective market information and provide first-hand information for company decision-making. Thus, SexyTea can provide better service and products to customers.

\subsection{Open Online Order}

Under the pandemic circumstances, the industry of tea drink is impacted for half of year. SexyTea has opened a new distribution online, which is based on takeaway app. Customers can order tea at home and the takeaways will deliver the order to your home. In order to ensure the quality of beverages, the distribution range of each store is three kilometers, and at the same time, it has established a strategic partnership with takeaway company to ensure delivery efficiency. The development of online sales channels for SexyTea will bring huge profit growth.

\section{CONCLUSION}

SexyTea always has an ambition to expand its branches to a wider market, such as Beijing, Shanghai and Shenzhen. But they did not have enough funds to compete with other giants yet, so they decided to expand influence and brand reputation at first. Therefore, they cleverly use special marketing strategies and gain the attention of their target customers. Creating a relationship with tourism not only enlarges the potential consumer groups but endues SexyTea a status of representative of one city. On the basis of good quality and humanized service, SexyTea uses social media to promote themselves and form a welcome brand image to increase customer loyalty. Finally, the high-density of the store in Changsha marks the high market share in this region. To minimize the impact of the pandemic, SexyTea opens online order system and gets considerable revenue. Since the takeaway industry is booming now, the future outlook of online order is positive.

Now, SexyTea has stepped out of Changsha city. They are welcomed by local consumers in Wuhan and Changde and consumers are willing to spend 7-8hour on a cup of tea. Therefore, the brand strategy of SexyTea is successful for now. If they want to compete with other giants in first-tier cities of China, they are required to have a transformative change in brand strategy.

\section{ACKNOWLEDGMENT}

In addition to my knowledge of marketing, the most important thing is to thank professor Noel Capon for the practical experience he has brought me in the course. And I tried to apply theoretical knowledge into practice, which was the biggest harvest and progress. And the TA Priscilla Jiang helped me to organize the structure of the paper. And many good teachers are proposing the mistake and give me advice, which is grateful.

\section{REFERENCES}

[1] He Qunxian. Talking about the tea concept innovation and quality control of new Chinese tea drinking[J]. China Tea, 2019, 41(02): 43-46. 
[2] 2020 White paper of New tea. CBNDATA.

https://www.cbndata.com/report/2486/detail?isRea ding $=$ report \&page $=1$

[3] Wu Yanjue, Wen Xingzi. Analysis on the Differential Marketing Strategy of my country's New Tea Brands Based on 4V Marketing Theory_-Taking "Sexy Tea" as an Example [J]. Market Modernization, 2020(17): 91-93.

[4] Wang Yingchang. 4P marketing strategy analysis of new-style tea drinks-taking SexyTea as an example[J]. Economist, 2020(07): 280-281.

[5] Shi Lin. A study on consumer psychology and behavior of new-style tea drinkers-Sentiment Analysis Based on Consumer Evaluation Language[J]. Gourmet Research, 2020, 37(02): 14-20.

[6] Definition of digital marketing. Financial Times. Archived from the original on 29 November 2017. Retrieved 22 August 2015.

[7] The Four Faces of Digital Marketing. American Marketing Association. Retrieved 22 August 2019.

[8] Liu Jing, Liu Xuan, Chen Min, Li Jiayi. Research on Enterprise New Media Marketing Strategy Based on Marketing 3.0 Perspective-Taking "Sexy Tea" as an Example [J]. Modern Commercial Industry, 2020, 41(01): 64-66.

[9] Brand Image, MSG.2020. https://www.managementstudyguide.com/brand-im age.htm. 